\title{
FRACTIONAL-ORDER UNIAXIAL VISCO-ELASTO-PLASTIC MODELS FOR STRUCTURAL ANALYSIS
}

\author{
J.L. Suzuki ${ }^{1}$, M. Zayernouri ${ }^{2}$, M.L. Bittencourt ${ }^{3}$, and G.E. Karniadakis ${ }^{4}$ \\ ${ }^{1}$ Department of Integrated System, Faculty of Mechanical Engineering, \\ University of Campinas, Campinas SP, 13083-970, Brazil \\ jlsuzuki@fem.unicamp.br \\ ${ }^{2}$ Division of Applied Mathematics, Brown University, Providence, RI 02912, USA \\ mohsen_zayernouri@brown.edu \\ http://www.dam.brown.edu/fpde/doku.php/mohsen_zayernouri \\ ${ }^{3}$ Department of Integrated System, Faculty of Mechanical Engineering, \\ University of Campinas, Campinas SP, 13083-970, Brazil \\ mlb@fem.unicamp.br \\ http://www.fem.unicamp.br \\ ${ }^{4}$ Division of Applied Mathematics, Brown University, Providence, RI 02912, USA \\ george_karniadakis@brown.edu \\ http://www.cfm.brown.edu/people/gk/
}

Key words: Fractional-order constitutive laws, nonlocal visco-plasticity, large strains, time-fractional integration.

Abstract. Three fractional-order models for uniaxial large strains and rate-dependent
plastic behavior of materials in structural analysis are proposed. Our approach is amenable
to modeling nonlinear and more sophisticated effects namely visco-elasto-plastic response
of materials. This approach seamlessly interpolates between the standard elasto-plastic
and visco-plastic models in plasticity, taking into account the history-dependency of
the accumulated plastic strain to specify the state of stress. To this end, we propose
three models namely i) viscoelasto-plastic with linear hardening plastic model, ii) elasto-
viscoplastic model, and iii) visco-elasto-plastic model, which combines the first the second
models. We employ a fractional-order constitutive law that relates the Kirchhoff stress
to its Caputo time-fractional derivative of order $\alpha \in(0,1]$. When $\alpha \rightarrow 0$ the standard
elasto-plastic (rate-independent) model and when $\alpha=1$, the corresponding visco-plastic
model is recovered. Since the material behavior is path-dependent the evolution of the
plastic strain is achieved by fractional-order time integration of the plastic strain rate with
respect to time. The strain rate is then obtained by means of the corresponding plastic
multiplier and deriving proper consistency conditions. Finally, we develop a so called 
fractional return-mapping algorithm for solving the nonlinear system of the equilibrium equations developing in each model.

\section{INTRODUCTION}

Fractional differential operators appear in many systems in science and engineering such as viscoelastic materials [1,2], electrochemical processes [3], porous or fractured media [4]. For instance, it has been found that the transport dynamics in complex and/or disordered systems is governed by non-exponential relaxation patterns $[5,6]$. For such processes, a time-fractional equation, in which the time-derivative emerges as $\mathcal{D}_{t}^{\nu} u(t)$, appears in the continuous limit. One interesting application of fractional calculus is to model (in a reduced fashion) complex elasto-plastic behavior of engineering materials (e.g. $[7,8]$ ) employing fractional-order models. Recently, fractional calculus has been employed as a novel tool for modelling material of heterogeneity/multi-scale effects to the constitutive model $[9,10]$, where the fractional viscoplasticity has been introduced as a generalization of classical Perzynas type viscoplasticity [11]. The fundamental role in the formulation plays the definition of the directions of a viscoplastic strains given as a fractional gradient of plastic potential. Formulating fast and accurate numerical methods for solving the resulting system of fractional ODEs/PDEs in such problems is the key to incorporate such nonlocal models in engineering applications.

Efficient discretization of the fractional operators is crucial. Lubich $[12,13]$ is the pioneer of the idea of discretized fractional calculus within the spirit of finite difference method (FDM). Later, Sanz-Serna [14] adopted the idea of Lubich and presented a temporal semi-discrete algorithm for partial integro-differential equations, which was first order accurate. Sugimoto [15] also employed a FDM for approximating the fractional derivative emerging in Burgers' equation. Later on, Gorenflo et. al. [16] adopted a finite-difference scheme by which they could generate discrete models of random walk in such anomalous diffusion. Diethelm et al. proposed a predictor-corrector scheme in addition to a fractional Adams method [17, 18]. After that, Langlands and Henry [19] considered the fractional diffusion equation, and analyzed the $L^{1}$ scheme for the time-fractional derivative. Sun and $\mathrm{Wu}[20]$ also constructed a difference scheme with $L^{\infty}$ approximation of time-fractional derivative. Of particular interest, Lin and $\mathrm{Xu}$ [21] analyzed a FDM for the discretization of the time-fractional diffusion equation with order $(2-\alpha)$. However, there are other class of global methods (spectral and spectral element methods) for discretizing fractional ODEs/PDEs (e.g., [22, 23, 24]), which are efficient for low-to-high dimensional problems.

The main contribution of the present work is to propose and solve three fractional-order models for uniaxial large deformation and rate-dependent plastic behavior of materials in structural analysis. This generalized framework is amenable to modeling nonlinear and more sophisticated effects namely visco-elasto-plastic response of materials. Furthermore, 
we develop a novel algorithm, called fractional return-mapping algorithm for solving the nonlinear system of the equilibrium equations developing in each model.

\section{DEFINITIONS}

We start with some preliminary definitions of fractional calculus [25]. The left-sided Riemann-Liouville integrals of order $\mu$, when $0<\mu<1$, is defined, as

$$
\left({ }_{x_{L}}^{R L} \mathcal{I}_{x}^{\mu} f\right)(x)=\frac{1}{\Gamma(\mu)} \int_{x_{L}}^{x} \frac{f(s) d s}{(x-s)^{1-\mu}}, \quad x>x_{L},
$$

where $\Gamma$ represents the Euler gamma function. The corresponding inverse operator, i.e., the left-sided fractional derivatives of order $\mu$, is then defined based on (1) as

$$
\left({ }_{x_{L}}^{R L} \mathcal{D}_{x}^{\mu} f\right)(x)=\frac{d}{d x}\left({ }_{x_{L}}^{R L} \mathcal{I}_{x}^{1-\mu} f\right)(x)=\frac{1}{\Gamma(1-\mu)} \frac{d}{d x} \int_{x_{L}}^{x} \frac{f(s) d s}{(x-s)^{\mu}}, \quad x>x_{L} .
$$

Furthermore, the corresponding left-sided Caputo derivatives of order $\mu \in(0,1)$ is obtained as

$$
\left({ }_{x_{L}}^{C} \mathcal{D}_{x}^{\mu} f\right)(x)=\left({ }_{x_{L}}^{R L} \mathcal{I}_{x}^{1-\mu} \frac{d f}{d x}\right)(x)=\frac{1}{\Gamma(1-\mu)} \int_{x_{L}}^{x} \frac{f^{\prime}(s) d s}{(x-s)^{\mu}}, \quad x>x_{L} .
$$

The definitions of Riemann-Liouville and Caputo derivatives are linked by the following relationship, which can be derived by a direct calculation

$$
\left({ }_{x_{L}}^{R L} \mathcal{D}_{x}^{\mu} f\right)(x)=\frac{f\left(x_{L}\right)}{\Gamma(1-\mu)\left(x+x_{L}\right)^{\mu}}+\left({ }_{x_{L}}^{C} \mathcal{D}_{x}^{\mu} f\right)(x),
$$

which denotes that the definition of the aforementioned derivatives coincide when dealing with homogeneous Dirichlet initial/boundary conditions.

\section{MATHEMATICAL MODELING}

We adopt a hierarchy approach and in three steps, we develop a novel framework, in which the history effects in elasto-plastic large strains in a mechanical member can be rigorously incorporated in computing the plastic strains. We present an efficient algorithm to solve the nonlinear system of fractional-order differential equations for the third model and remark how it recovers the first two models.

First, we consider a viscoelastic material with a linear hardening model, shown in Fig. 1 (top). In this model, we assume that the total strain $\varepsilon$ splits into a viscoelastic part $\varepsilon^{v e}$ on the Scott-Blair element with constant $E$ and the fractional order $\beta$, and a plastic strain $\varepsilon^{p}$. The fractional-order Scott-Blair element in fact interpolates between a perfect linear elastic spring when $\beta=0$ and an ideal Newtonian viscous element if $\beta=1$. The second model considers an elastic-viscoplastic material with nonlinear hardening, shown 
in Fig. 1 (middle). In this case, the total strain $\varepsilon$ is split into an elastic strain $\varepsilon^{e}$ for the elastic spring with constant $E$ and a viscoplastic strain $\varepsilon^{v p}$ on the Scott-Blair element with constant $K_{f}$ and fractional order $\beta_{f}$. Therefore the Scott-Blair element interpolates between a linear hardening spring and an ideal Newtonian viscous element. The third model combines the first two, thus with viscoelastic strain $\varepsilon^{v e}$ for the Scott-Blair element with constant $E$ and fractional order $\beta$ and viscoplastic strain $\varepsilon^{v p}$ for the Scott-Blair element with constant $K_{f}$ with fractional order $\beta_{f}$.
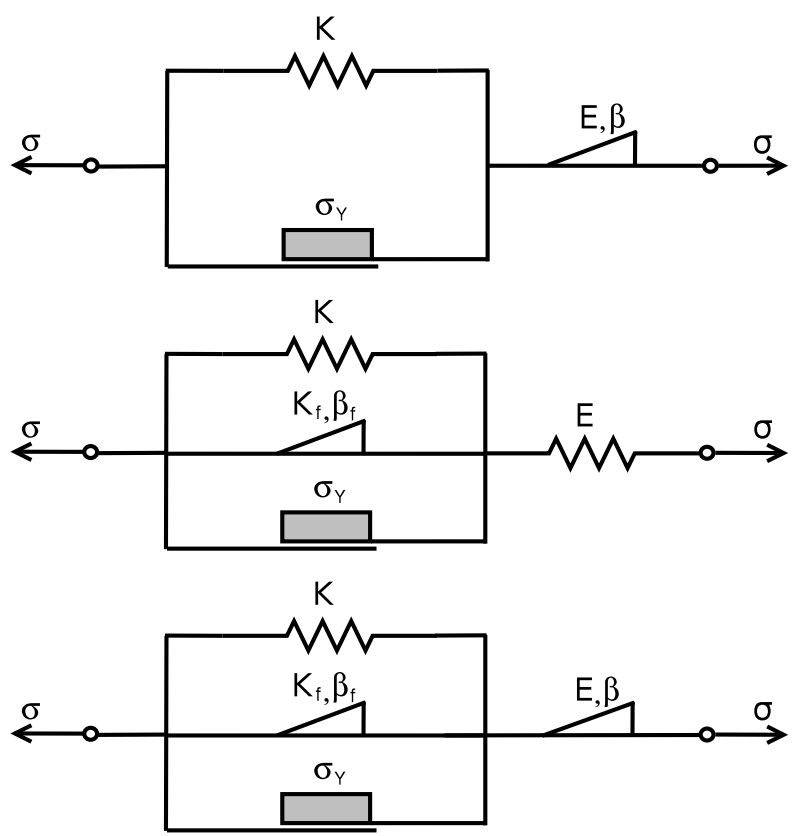

Figure 1: (top): viscoelasto-plastic model with standard linear hardening model, (middle): elasto-viscoplastic model, (bottom): visco-elasto-plastic model.

\subsection{Rate-Dependent Visco-Elasto-Plastic Model with Nonlinear Hardening}

The nonlocal constitutive law for this model is assumed to be of the form:

$$
\begin{aligned}
\sigma & =E{ }_{0}^{C} \mathcal{D}_{t}^{\beta} \varepsilon^{v e}(t) \\
& =E{ }_{0}^{C} \mathcal{D}_{t}^{\beta}\left(\varepsilon-\varepsilon^{v p}\right) .
\end{aligned}
$$

To designate a set of admissible stresses, we define the the following closed convex stress space:

$$
\mathbb{E}_{\sigma}=\{\sigma \in \mathbb{R} \mid f(\sigma, \alpha) \leq 0\},
$$

where $f: \mathbb{R} \times \mathbb{R}^{+} \rightarrow \mathbb{R}$ represents the yield condition, given by:

$$
f(\sigma, \alpha)=|\sigma|-\left[\sigma_{Y}+K \alpha+K_{f}{ }_{0}^{C} \mathcal{D}_{t}^{\beta_{f}}(\alpha)\right] .
$$


The corresponding boundary of $\mathbb{E}_{\sigma}$ is a convex set $\partial \mathbb{E}_{\sigma}$, given as

$$
\partial \mathbb{E}_{\sigma}=\{\sigma \in \mathbb{R} \mid f(\sigma, \alpha)=0\} .
$$

In this model, we assume that the hardening is isotropic in the sense that at any state of loading the center of $\mathbb{E}_{\sigma}$ remains at the origin, moreover, the hardening is nonlinear in the amount of plastic flow.

\subsection{Incremental Strain}

In this section we present the nonlocal backward-Euler time integration procedure for model 3, based on time integration procedures for plasticity, where a trial state is defined by freezing the internal variables. However, in this case we have a fractional returnmapping algorithm, where the solution for the plastic slip is now given by a fractional order differential equation.

\subsubsection{Fractional return-mapping algorithm}

$\mathbf{i}$ - Database at $x \in \Omega:\left\{\varepsilon_{n}^{v p}, \alpha_{n}, \Delta \gamma_{n}\right\}$

ii - Impose a strain increment $\Delta \varepsilon_{n}$

$$
\varepsilon_{n+1}=\varepsilon_{n}+\Delta \varepsilon_{n}
$$

iii - Trial state (freezing the viscoplastic state):

$$
\begin{gathered}
\varepsilon_{n+1}^{v p^{\text {trial }}}=\varepsilon_{n}^{v p}, \quad \alpha_{n+1}^{\text {trial }}=\alpha_{n} \\
\sigma_{n+1}^{\text {trial }}=E{ }_{0}^{C} \mathcal{D}_{t}^{\beta}\left(\varepsilon_{n+1}-\varepsilon_{n}^{v p}\right) \\
f_{n+1}^{\text {trial }}=\left|\sigma_{n+1}^{\text {trial }}\right|-\left[\sigma_{Y}+K \alpha_{n}+K_{f}{ }_{0}^{C} \mathcal{D}_{t}^{\beta_{f}} \alpha_{n}\right]
\end{gathered}
$$

If $f_{n+1}^{\text {trial }} \leq 0$ then viscoelastic state

$$
\varepsilon_{n+1}^{v p}=\varepsilon_{n}^{v p}, \quad \alpha_{n+1}=\alpha_{n}, \quad \sigma_{n+1}=\sigma_{n+1}^{\text {trial }}
$$

Else: viscoplastic state

We require that $f\left(\sigma_{n+1}, \alpha_{n+1}\right)=0$, which translates into the following FODE:

$$
E_{0}^{C} \mathcal{D}_{t}^{\beta}(\Delta \gamma)+K_{f}{ }_{0}^{C} \mathcal{D}_{t}^{\beta_{f}}(\Delta \gamma)+K \Delta \gamma=f_{n+1}^{\text {trial }}
$$

After solving for $\Delta \gamma$, we update:

$$
\begin{gathered}
\varepsilon_{n+1}^{v p}=\varepsilon_{n}^{v p}+\Delta \gamma \operatorname{sign}\left(\sigma_{n+1}^{\text {trial }}\right) \\
\alpha_{n+1}=\alpha_{n}+\Delta \gamma \\
\sigma_{n+1}=\sigma_{n+1}^{\text {trial }}-E{ }_{0}^{C} \mathcal{D}_{t}^{\beta}[\Delta \gamma] \operatorname{sign}\left(\sigma_{n+1}^{\text {trial }}\right)
\end{gathered}
$$


We remark that by setting $K_{f}=0$ in Eqs. 12 and 14 we recover model 1 , and model 2 is recovered when $\beta \rightarrow 0$. Furthermore, when $K_{f}=0$ and $\beta \rightarrow 0$ we recover rateindependent elastoplasticity with linear isotropic hardening.

The fractional derivatives present in the modified return-mapping procedure are computed implicitly using the finite difference method (FDM) developed in [21], where ${ }_{0} \mathcal{D}_{t}^{\nu} u(t)$ is represented as

$$
{ }_{0} \mathcal{D}_{t}^{\nu} u(t)=\frac{1}{\Gamma(2-\nu)} \sum_{j=0}^{k} b_{j} \frac{u\left(t_{k+1-j}\right)-u\left(t_{k-j}\right)}{(\Delta t)^{\nu}}+r_{\Delta t}^{k+1}
$$

where $r_{\Delta t}^{k+1} \leq C_{u}(\Delta t)^{2-\nu}$ and $b_{j}:=(j+1)^{1-\nu}-j^{1-\nu}, j=0,1, \cdots, k$.

\section{COMPUTATIONAL RESULTS}

In order to show the influence of the fractional order parameters $\beta$ and $\beta_{f}$ on the stress response, we performed monotonic and cyclic tests at different strain rates. The purpose is to show the recovery of rate-independent elastoplasticity with isotropic hardening when $\beta$ and $\beta_{f}$ are close to zero (e.g. $\beta=\beta_{f}=0.01$ ), and viscoelastic and viscoplastic behaviors when $\beta, \beta_{f}$ approach 1 .

The material properties used for all models are $E=100, \sigma_{Y}=1$. For rate-independent elastoplasticity and model 1 we consider $K=1$. For models 2 and 3 we used $K=0$ and $K_{f}=1$, to make the evolution of the internal hardening variable $\alpha$ dependent only on the Caputo derivative of Eq. 7.

\subsection{Monotonic strains}

For this test, we apply incremental prescribed strains until $\varepsilon=0.4$. We consider two distinct strain rates, with respective time parameters presented in Table 1.

Table 1: Time discretization parameters for the monotonic strains.

\begin{tabular}{cccc}
\hline Strain rate $\dot{\varepsilon}\left[s^{-1}\right]$ & Total time $T[s]$ & Number of increments $N$ & Time increment $\Delta t[s]$ \\
\hline 0.005 & 80 & 1000 & 0.08 \\
\hline 0.05 & 8 & 500 & 0.016 \\
\hline
\end{tabular}

The results for stress using model 1 are shown in Fig. 2 with several values for $\beta$. We note that the effect of increasing viscosity with the increase of $\beta$ is clear for both strain rates and the solution becomes rate-independent as $\beta \rightarrow 0$. The observed change in slope in the plastic region occurs because of the application of the fractional derivative in the constitutive equation, which involves the total and the plastic strains. The results obtained for model 2 are shown in Fig. 3. We recover the same results of rate-independentelastoplasticity when $\beta_{f}=0.01$. Also, the viscoplastic range becomes more sensitive to rate-dependent strains as we increase $\beta_{f}$. 
A more interesting behavior is presented in Fig. 4 for model 3 when $\beta=\beta_{f}$. The results obtained for $\beta=\beta_{f}=0.99$ are the same as model 1 , because in this case the Scott-Blair element behaves as a Newton viscous element, which is too dissipative to surpass the yield stress for lower strain rates. Generally, model 3 recovers model 1 when $\beta_{f} \rightarrow 0$, while model 2 is recovered if $\beta \rightarrow 0$.

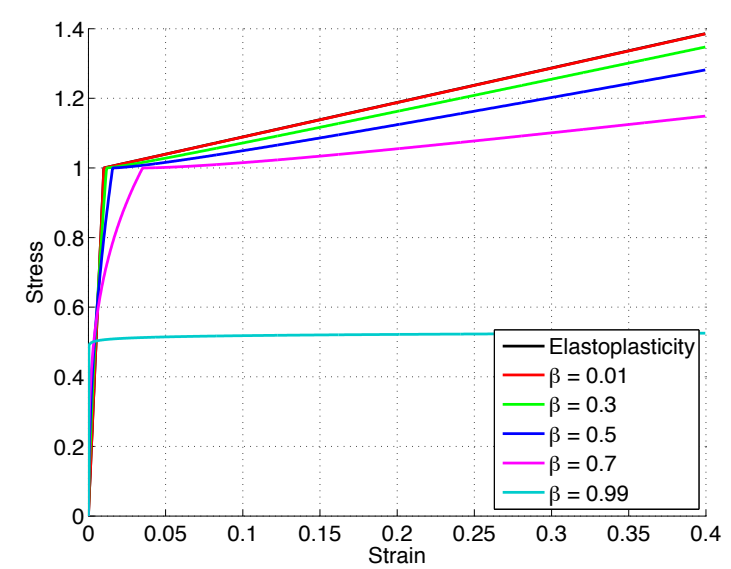

(a) $\dot{\varepsilon}=0.005 \mathrm{~s}^{-1}$

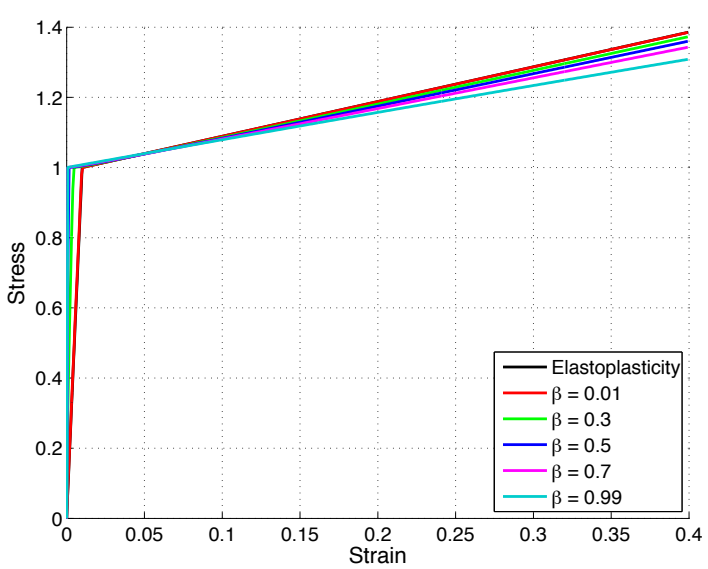

(b) $\dot{\varepsilon}=0.05 \mathrm{~s}^{-1}$

Figure 2: Stress versus strain response for model 1. We observe the recovery of the rateindependent solution when $\beta=0.01$. The variation of $\beta$ has effect on the viscoelastic response which influences the strain threshold for plasticity and also the slope of the curve in the plastic region. 


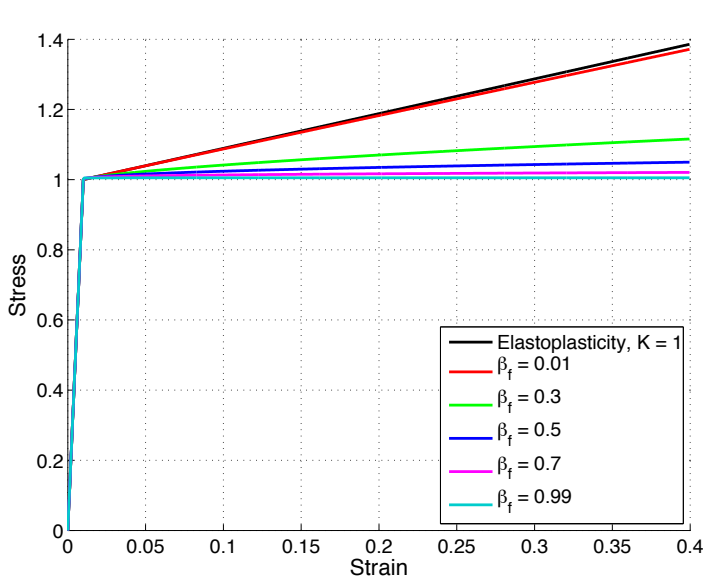

(a) $\dot{\varepsilon}=0.005 \mathrm{~s}^{-1}$

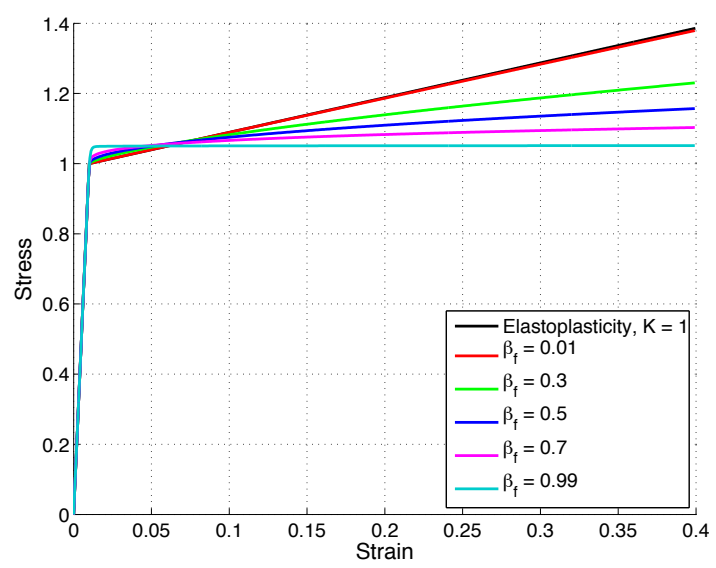

(b) $\dot{\varepsilon}=0.05 \mathrm{~s}^{-1}$

Figure 3: Stress versus strain response for model 2. We observe the rate-independent behavior when $\beta_{f}=0.01$ and the nonlinear behavior in viscoplasticity as we increase the value of $\beta$ for a higher strain rate in (b). Because we chose $K=0$, when $\beta_{f}=0.99$ the fractional element behaves as a Newton element, which recovers perfect viscoplasticity when the strain rates are sufficiently small (a).

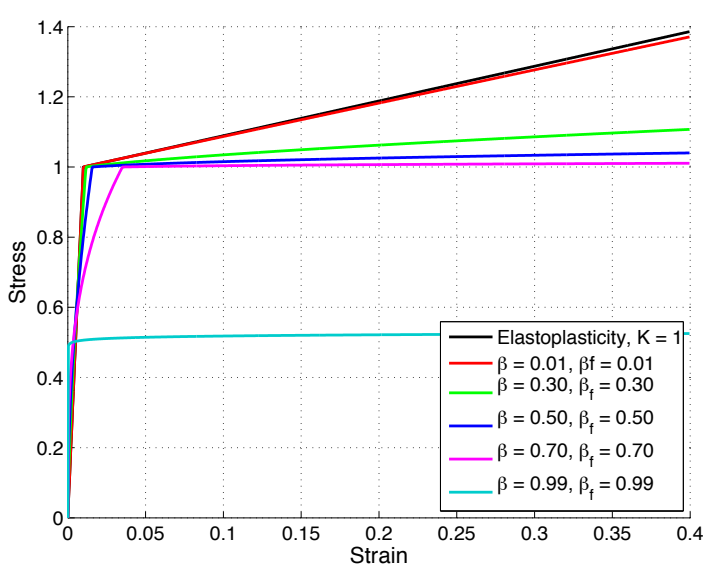

(a) $\dot{\varepsilon}=0.005 \mathrm{~s}^{-1}$

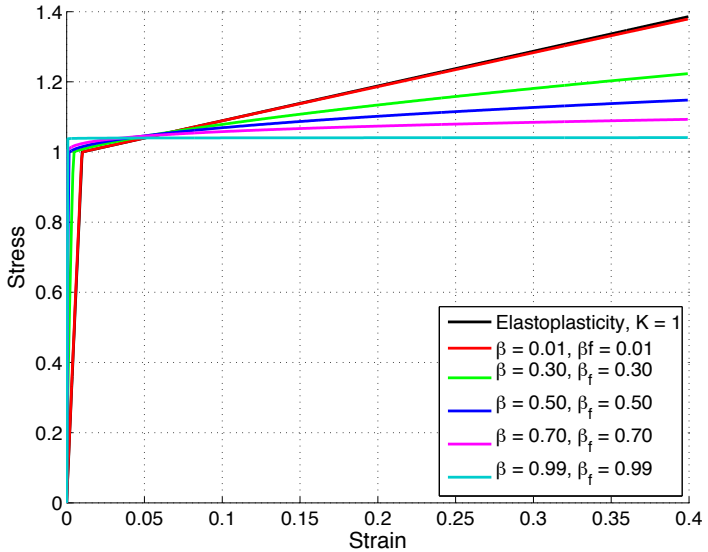

(b) $\dot{\varepsilon}=0.05 \mathrm{~s}^{-1}$

Figure 4: Stress versus strain response for model 3 with $\beta=\beta_{f}$. We observe a combined behavior of models 1 and 2, constituting visco-elasto-plastic behavior. In this case, the viscoplastic range is affected by both fractional-order parameters. We also observe the significant change in behavior of the stress from (a) to (b) when $\beta=\beta_{f}=0.99$. 


\subsection{Cyclic strains}

To compute the loading/unloading response of the models and analyze the hardening behavior, we perform a cyclic test comprising three loading cycles with incremental strains. We start with a traction cyclic from $\varepsilon=0$ to $\varepsilon=0.4$, followed by unloading and compression cycle until $\varepsilon=-0.4$, from where we increment the strains again up to $\varepsilon=0.4$. We consider the same strain rates as the case of monotonic loading, with time parameters presented in Table 2 .

Table 2: Time discretization parameters for the cyclic strains.

\begin{tabular}{cccc}
\hline Strain rate $\dot{\varepsilon}\left[s^{-1}\right]$ & Total time $T[s]$ & Number of increments $N$ & Time increment $\Delta t[s]$ \\
\hline 0.005 & 400 & 5000 & 0.08 \\
\hline 0.05 & 40 & 10000 & 0.004 \\
\hline
\end{tabular}

The results for the cyclic tests are presented in Figs. 5-7. All models recover the rateindependent behavior when the order of the fractional derivative gets sufficiently close to zero. It is important to note for all models, despite the lower stress levels of $\beta=\beta_{f}=0.7$ compared to $\beta=\beta_{f}=0.01$ obtained for the applied strain rates, the inverse situation may occur if the strain rates are sufficiently high.

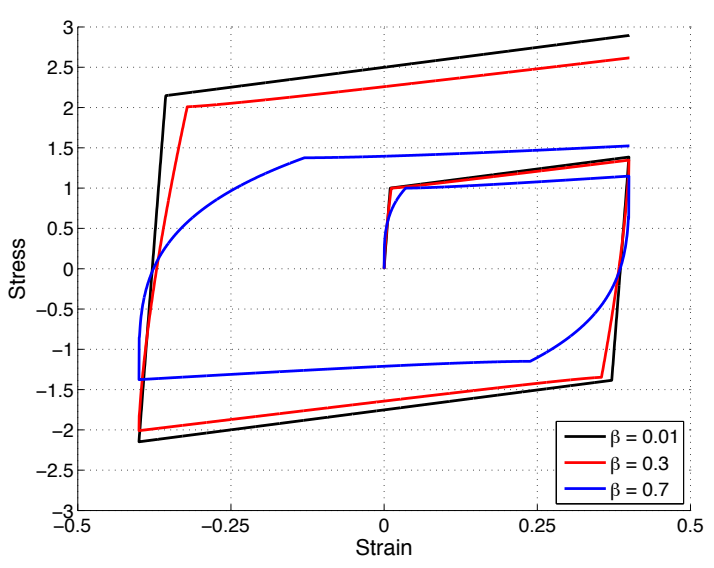

(a) $\dot{\varepsilon}=0.005 \mathrm{~s}^{-1}$

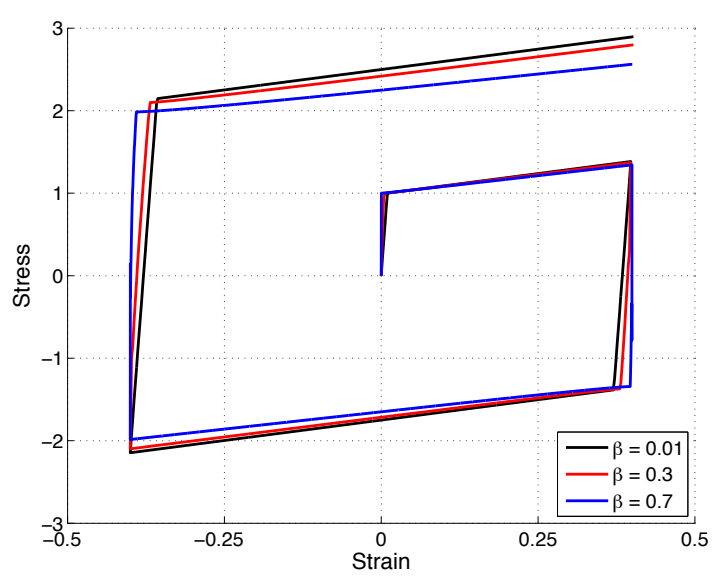

(b) $\dot{\varepsilon}=0.05 \mathrm{~s}^{-1}$

Figure 5: Cyclic stress versus strain response for model 1 with different values of $\beta$. We observe the inelastic loading/unloading behavior. The use of higher fractional order values like $\beta=0.7$ led to increased stiffness in the viscoelastic region, according to the applied strain rate. However, the influence of the fractional derivative on the plastic strains led to less hardening at the end of the cycle when compared to smaller values of $\beta$. 


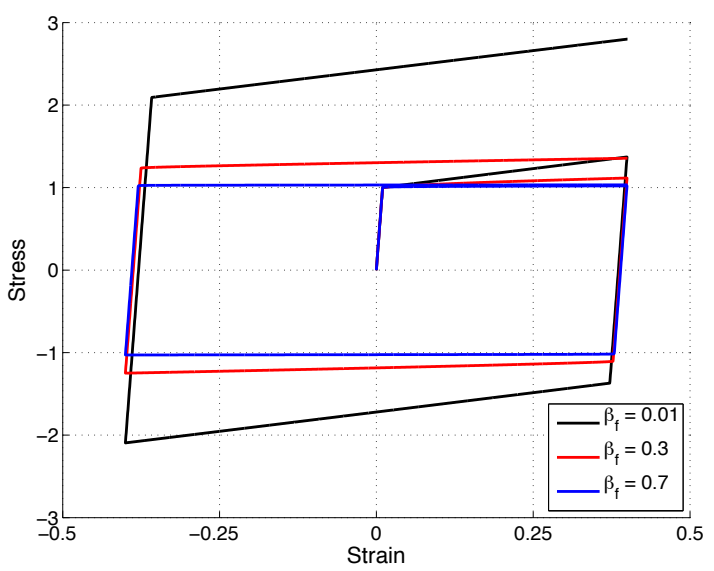

(a) $\dot{\varepsilon}=0.005 \mathrm{~s}^{-1}$

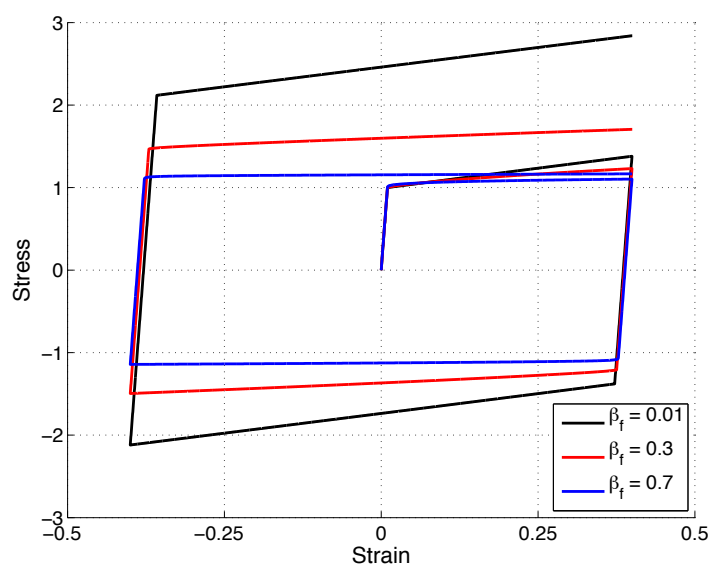

(b) $\dot{\varepsilon}=0.05 \mathrm{~s}^{-1}$

Figure 6: Cyclic stress versus strain response for model 2 with different values of $\beta$. The loading/unloading behavior is linear. We observe that when we increase $\beta_{f}$ the cyclic stress response presents less hardening for the applied strain rates.

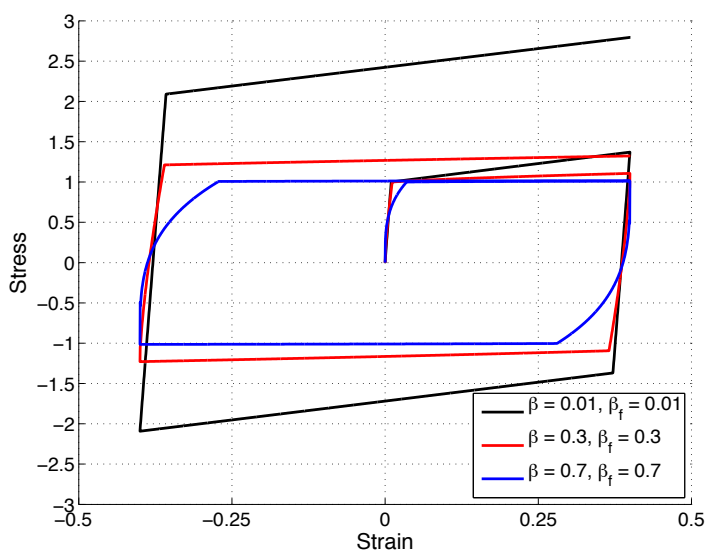

(a) $\dot{\varepsilon}=0.005 \mathrm{~s}^{-1}$

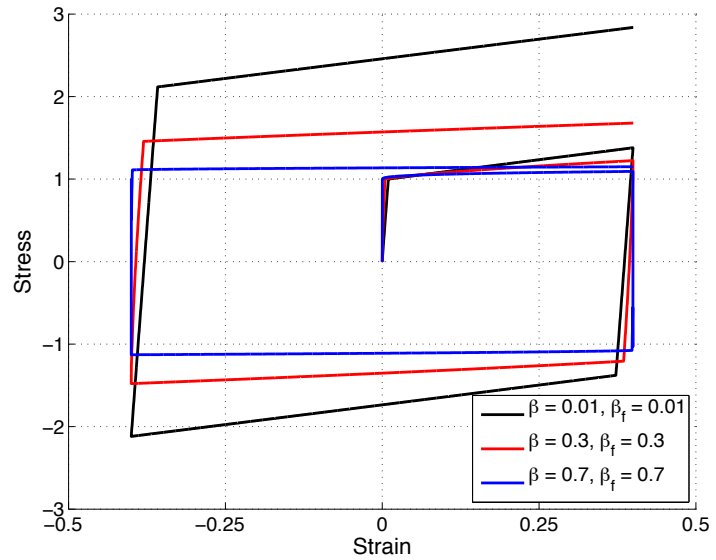

(b) $\dot{\varepsilon}=0.05 \mathrm{~s}^{-1}$

Figure 7: Cyclic stress versus strain response for model 3 with $\beta=\beta_{f}$. We observe the inelastic loading/unloading response with the same viscoplastic effects as observed from the cyclic response of model 2 .

\section{CONCLUSION}

The main contribution of the present work was to propose three fractional-order models for uniaxial large strains and rate-dependent plastic behavior of materials in structural analysis. This generalized framework is amenable to modeling nonlinear and more sophis- 
ticated effects namely visco-elasto-plastic response of materials. A novel algorithm, called fractional return-mapping algorithm was proposed to solve nonlinear system of equilibrium equations developing in the third model. The recovery of the incremental equations to the other models was shown using the appropriate fractional-order parameters. All proposed models recover rate-independent plasticity when $\beta, \beta_{f} \rightarrow 0$. Also, the third model generalized the first two models. According to the choice of material parameters $K, K_{f}$ and the fractional orders $\beta, \beta_{f}$, models 2 and 3 can recover viscous Newtonian elements with or without hardening, and also the mixed spring-dashpot behavior of the Scott-Blair element. The formulation presented in this work can be added to a FEM planar or three-dimensional truss code in a straightforward way and simulate the behavior of time-dependent large inelastic strains.

\section{REFERENCES}

[1] F. Mainardi, Fractional calculus and waves in linear viscoelasticity: an introduction to mathematical models, Imperial College Press, 2010.

[2] B. J. West, M. Bologna, P. Grigolini, Physics of Fractal Operators, New York, NY: Springer Verlag., 2003.

[3] M. Ichise, Y. Nagayanagi, T. Kojima, An analog simulation of non-integer order transfer functions for analysis of electrode processes, Journal of Electroanalytical Chemistry and Interfacial Electrochemistry 33 (2) (1971) 253-265.

[4] D. A. Benson, S. W. Wheatcraft, M. M. Meerschaert, Application of a fractional advection-dispersion equation, Water Resources Research 36 (6) (2000) 1403-1412.

[5] R. Metzler, J. Klafter, The random walk's guide to anomalous diffusion: a fractional dynamics approach, Physics reports 339 (1) (2000) 1-77.

[6] R. Klages, G. Radons, I. M. Sokolov, Anomalous Transport: Foundations and Applications, Wiley-VCH, 2008.

[7] J. Bonet, R. Wood, Nonlinear continuum mechanics for finite element analysis, Cambridge.

[8] J. Simo, T. Hughes, Computational Inelasticity, Springer-Verlag, 1998.

[9] W. Sumelka, Fractional viscoplasticity, in: 37th Solid Mechanics Conference, Warsaw, Poland, 2012, p. 102103.

[10] W. Sumelka, Fractional viscoplasticity: an introduction, in: Workshop 2012- Dynamic Behavior of Materials and Safety of Structures, Poznan, Poland, 2012, pp. $1-2$. 
[11] P. Perzyna, The constitutive equations for rate sensitive plastic materials, Tech. rep., DTIC Document (1962).

[12] C. Lubich, On the stability of linear multistep methods for volterra convolution equations, IMA Journal of Numerical Analysis 3 (4) (1983) 439-465.

[13] C. Lubich, Discretized fractional calculus, SIAM Journal on Mathematical Analysis 17 (3) (1986) 704-719.

[14] J. Sanz-Serna, A numerical method for a partial integro-differential equation, SIAM journal on numerical analysis 25 (2) (1988) 319-327.

[15] N. Sugimoto, Burgers equation with a fractional derivative; hereditary effects on nonlinear acoustic waves, J. Fluid Mech 225 (631-653) (1991) 4.

[16] R. Gorenflo, F. Mainardi, D. Moretti, P. Paradisi, Time fractional diffusion: a discrete random walk approach, Nonlinear Dynamics 29 (1-4) (2002) 129-143.

[17] K. Diethelm, N. J. Ford, Analysis of fractional differential equations, Journal of Mathematical Analysis and Applications 265 (2) (2002) 229-248.

[18] K. Diethelm, N. J. Ford, A. D. Freed, Detailed error analysis for a fractional adams method, Numerical algorithms 36 (1) (2004) 31-52.

[19] T. Langlands, B. Henry, The accuracy and stability of an implicit solution method for the fractional diffusion equation, Journal of Computational Physics 205 (2) (2005) $719-736$.

[20] Z.-z. Sun, X. Wu, A fully discrete difference scheme for a diffusion-wave system, Applied Numerical Mathematics 56 (2) (2006) 193-209.

[21] Y. Lin, C. Xu, Finite difference/spectral approximations for the time-fractional diffusion equation, Journal of Computational Physics 225 (2) (2007) 1533-1552.

[22] M. Zayernouri, G. E. Karniadakis, Exponentially accurate spectral and spectral element methods for fractional odes, J. Comp. Physics 257 (2014) 460-480.

[23] M. Zayernouri, M. Ainsworth, G. E. Karniadakis, A unified Petrov-Galerkin spectral method for fractional PDEs, Computer Methods in Applied Mechanics and Engineering 283 (2015) 1545-1569.

[24] M. Zayernouri, G. E. Karniadakis, Fractional spectral collocation methods for linear and nonlinear variable order FPDEs, Journal of Computational Physics (Special issue on FPDEs: Theory, Numerics, and Applications) 293 (2015) 312-338.

[25] I. Podlubny, Fractional Differential Equations, San Diego, CA, USA: Academic Press, 1999. 